\title{
A study on bacteriological profile and antimicrobial resistance pattern from various body fluids of patients attending the tertiary care Hospital, KIMS, Hubli
}

\author{
Harshika Y K ${ }^{1}$, Shobha Medegar K. $\mathbf{R}^{2, *}$, Asha B Patil ${ }^{3}$,Smita N R \\ ${ }^{1,2}$ Tutor, ${ }^{3}$ Professor and Head, ${ }^{4}$ Post Graduate, Dept. of Microbiology, Karnataka Institute of Medical Sciences, Hubli, \\ Karnataka, India
}

*Corresponding Author: Shobha Medegar K. R

Email: drshobha83m@gmail.com

\begin{abstract}
Introduction: Body fluids like pleural fluid, ascitic fluid, cerebrospinal fluid etc are usually sterile, but they can get infected by different micro organisms, thus leading to life threatening infections. ${ }^{1,2}$

Aims and Objectives: 1) To isolate the causative organisms of sterile body fluid infections. 2) To determine the antimicrobial resistance pattern of the isolates.

Materials and Methods: 1) This is a retrospective observational study, conducted from January 2017 to December 2017 in the department of Microbiology KIMS Hubli. 2) Sterile body fluids were processed according to Standard laboratory Procedures. 3) Antimicrobial susceptibility testing was performed by Kirby-Bauer disc diffusion method as per CLSI guidelines.

Results: A total of 635 samples were studied out of which $233(36.6 \%)$ were pleural fluids, $222(34.9 \%)$ were ascitic fluids, $174(27.4 \%)$ were cerebrospinal fluids and 06(0.9\%) were pericardial fluid. In our study the predominant organisms were E-coli (23.23\%) and NFGNB (19.01\%) followed by Pseudomonas (14.08\%), Klebsiellaspp (13.38\%), S.aureus (10.56\%) and Citrobacter spp (7.04\%). Gram negative isolates were 100\% sensitive to Imipenem followed by Amikacin (78\%), Gentamicin (74\%), Cefepime (69\%). The Gram positive isolates were 100\% sensitive to Vancomycin and Linezolid followed by Gentamicin (96\%), Cefepime (94\%), Amikacin (92\%). The Pseudomonas isolates were highly sensitive to Imipenem (96\%) and Piperacillin Tazobactum (92\%). High resistance was observed to Ceftazidime, Cefoperazone and Levofloxacin.

Conclusion: Knowledge of bacteriological and antimicrobial profile of sterile body fluids is necessary, so that the life threatening infections can be effectively treated and thus to prevent antimicrobial resistance associated with it.
\end{abstract}

Keywords: Sterile Body fluids, Antimicrobial resistance, E-coli, NFGNB, Kirby-Bauer disk diffusion method.

\section{Introduction}

Body fluids like pleural fluid, peritoneal fluid, CSF, synovial fluid and pericardial fluid are usually sterile. There are certain common pathogenic bacteria like E coli, Klebsiella species, Haemophilus influenza, Staphylococcus aureus, Neisseria Meningitidis, NFGNB (Non fermenting Gram Negative Bacillus), Pseudomonas, Acinetobacter, which invade and infect the sterile body fluids leading to morbidity and life threatening infections. ${ }^{1,2}$

Hence infections of sterile body fluids are a medical emergency and needs an early diagnosis and effective treatment. Moreover for the better management of patients and framing the antibiotic policy, the knowledge of prevalent strains along with their antimicrobial resistant pattern is essential.

As of now, there are very limited data on bacterial profiles and their antimicrobial susceptibility pattern from body fluids in our geographical area. Hence assessing bacterial profiles and antimicrobial sensitivity pattern from body fluids is very crucial to clinicians, Microbiologists, Pharmacists and Policy makers for proper diagnosis of different infections and for prudent antibiotic use.

So the present study was undertaken to know the current status of bacterial profile and their susceptibility patterns from various body fluids collected from patients attending our tertiary care hospital.

\section{Materials and Methods}

This retrospective observational study was conducted between January 2017 to December 2017 in the department of Microbiology KIMS Hubli.

A total of 635 samples were analysed. Body fluid samples like Pleural, Peritoneal, Synovial, Pericardial and CSF were collected under proper aseptic precautions and processed within 2 hour of collection.

Inclusion Criteria: 1) All the samples received from patients with body fluid infections, admitted in KIMS hospital Hubli, irrespective of age and sex were included.

Exclusion Criteria: 1) Blood samples 2) Patients with history of antibiotics within the last 2 weeks 3) Contaminated samples 4) Delayed body fluids for more than 2 hours.

Sample Processing: The samples collected were processed in our laboratory using Standard microbiological procedures. The samples were subjected for Gram Stain and Culture. The culture media used were Chocolate Agar, Mac Conkey Agar and Thioglycollate Medium (Hi-Media, Mumbai India) to obtain isolated colonies. The isolated colonies were then identified by Gram Stain and Standard Biochemical tests.

Antimicrobial Susceptibility Test: The Antimicrobial sensitivity test was performed for isolated organisms by Kirby Bauer Disc Diffusion method according to CLSI guidelines. $^{7}$ 
Drugs for Gram Positive Cocci: Cefoxitin, Ciprofloxacin, Gentamicin, Cefotaxime, Cefepime, Tetracycline, Erythromycin, Clindamycin, Co trimoxazole, Amoxycillin Clavulanate, Linezolid and Levofloxacin, Vancomycin.

Drugs for Gram Negative Bacilli: Ampicillin, Amoxyclav, Cefotaxime, Ceftriaxone, Cefepime, Ceftazidime, Amikacin, Gentamicin, Imipenem, Levofloxacin, Cotrimoxazole.

Drugs for Pseudomonas: Piperacillin, Piperacillin tazobactum, Ceftazidime, Cefepime, Cefpodoxime, Cefeperazone, Amikacin, Gentamicin, Ciprofloxacin, Levofloxacin, Imipenem, Aztreonam.

\section{Results}

A total of 635 samples were collected from suspected patients which included pleural fluid, peritoneal fluid, Cerebrospinal fluid (CSF) and pericardial fluid. Out of 635 samples processed, 142 fluids showed growth with isolation rate of $22 \%$. The most common received fluid was pleural fluid (36.6\%), followed by Ascitic fluid (34.9\%), Cerebrospinal fluid (27.4\%) and Pericardial fluid (0.9\%).
Out of 142 culture positive samples, the predominant organism isolated was Escherichia coli (23.2\%), followed by NFGNB (19\%) and Pseudomonas (14\%), Klebsiella (13.4\%), Staphylococcus aureus (10.5\%). Less commonly isolated were Enterococcus species (2\%), Enterobacter (1.4\%), and Streptococcus pyogenes $(0.7 \%)$.

The Gram negative bacterial isolates showed multidrug resistance pattern (MDR) but they were $100 \%$ sensitive to Imipenem. They showed high resistance to Cephalosporins like ceftriaxone and cefotaxime and also to Amoxyclav, cotrimoxazole and Ampicillin.

Multi drug resistance pattern was not much observed in Gram positive isolates compared to Gram Negative isolates. They were relatively resistant to Tetracycline, Erythromycin, Clindamycin and Ampicillin. They were $100 \%$ sensitive to Vancomycin and Linezolid.

The Pseudomonas isolates showed high resistance to Cephalosporins and fluoroquinolones. They were highly sensitive to Imipenem (96\%) and Piperacillin tazobactam (92\%).

Table 1: Growth pattern of various body fluids

\begin{tabular}{|l|c|c|c|}
\hline & Total number of samples & Growth & No Growth \\
\hline Pleural fluid & 233 & 59 & 174 \\
\hline Ascitic fluid & 222 & 56 & 166 \\
\hline Cerebrospinal fluid & 174 & 23 & 151 \\
\hline Pericardial fluid & 06 & 04 & 02 \\
\hline Total & 635 & 142 & 493 \\
\hline
\end{tabular}

Table 2: Bacterial profile in different body fluid samples

\begin{tabular}{|l|c|c|c|c|c|}
\hline \multicolumn{1}{|c|}{ Organisms } & $\begin{array}{c}\text { Total } \\
\text { Number }\end{array}$ & Pleural fluid & $\begin{array}{c}\text { Ascitic } \\
\text { fluid }\end{array}$ & $\begin{array}{c}\text { Cerebro } \\
\text { Spinal Fluid }\end{array}$ & $\begin{array}{c}\text { Pericardial } \\
\text { fluid }\end{array}$ \\
\hline E-coli & 33 & 16 & 09 & 06 & 01 \\
\hline Klebsiella species & 21 & 06 & 09 & 06 & 01 \\
\hline NFGNB & 27 & 10 & 13 & 03 & 01 \\
\hline Pseudomonas aeruginosa & 20 & 09 & 09 & 02 & -- \\
\hline Citrobacter species & 13 & 06 & 05 & 01 & -- \\
\hline Staphylococcus aureus & 15 & 10 & 03 & 02 & -- \\
\hline CONS & 08 & 02 & 05 & 01 & 01 \\
\hline Enterobacter species & 02 & 01 & -- & -- & -- \\
\hline Streptococcus pyogenes & 01 & 00 & & -- & -- \\
\hline Enterococcus species & 03 & 03 & -- & -- & -- \\
\hline
\end{tabular}

Table 3: Antimicrobial sensitivity pattern of Gram negative bacterial (GNB) isolates. (n=94)

\begin{tabular}{|l|c|c|c|c|c|}
\hline \multicolumn{1}{|c|}{ Drugs } & $\begin{array}{c}\text { Escherichia } \\
\text { coli }\end{array}$ & $\begin{array}{c}\text { Klebsiella } \\
\text { species }\end{array}$ & NFGNB & $\begin{array}{c}\text { Citrobacter } \\
\text { species }\end{array}$ & $\begin{array}{c}\text { Enterobacter } \\
\text { species }\end{array}$ \\
\hline Ampicillin & $9 \%$ & $0 \%$ & $30 \%$ & $22 \%$ & $100 \%$ \\
\hline Amoxyclav & $44 \%$ & $44 \%$ & $63 \%$ & $64 \%$ & $100 \%$ \\
\hline Amikacin & $62 \%$ & $84 \%$ & $74 \%$ & $92 \%$ & $100 \%$ \\
\hline Gentamicin & $58 \%$ & $78 \%$ & $66 \%$ & $92 \%$ & $100 \%$ \\
\hline Ciprofloxacin & $62 \%$ & $56 \%$ & $70 \%$ & $86 \%$ & $100 \%$ \\
\hline Levofloxacin & $56 \%$ & $44 \%$ & $56 \%$ & $100 \%$ & $100 \%$ \\
\hline Cefotaxime & $18 \%$ & $34 \%$ & $58 \%$ & $58 \%$ & $0 \%$ \\
\hline Ceftriaxone & $18 \%$ & $38 \%$ & $58 \%$ & $58 \%$ & $0 \%$ \\
\hline
\end{tabular}




\begin{tabular}{|l|l|l|l|l|l|}
\hline Cefepime & $44 \%$ & $72 \%$ & $82 \%$ & $78 \%$ & $0 \%$ \\
\hline Cotrimoxazole & $44 \%$ & $34 \%$ & $60 \%$ & $42 \%$ & $0 \%$ \\
\hline Imipenem & $100 \%$ & $100 \%$ & $100 \%$ & $100 \%$ & $100 \%$ \\
\hline
\end{tabular}

Table 4: Antibiotic sensitivity pattern of Gram positive isolates $(n=28)$

\begin{tabular}{|l|c|c|c|c|}
\hline \multicolumn{1}{|c|}{ Drugs } & $\begin{array}{c}\text { Staphylococcus } \\
\text { aureus }\end{array}$ & $\begin{array}{c}\text { CONS (Coagulase negative } \\
\text { Staphylococcus) }\end{array}$ & $\begin{array}{c}\text { Enterococcus } \\
\text { Species }\end{array}$ & $\begin{array}{c}\text { Streptococcus } \\
\text { pyogenes }\end{array}$ \\
\hline Ampicillin & $16 \%$ & $33 \%$ & $0 \%$ & $0 \%$ \\
\hline Amoxyclav & $84 \%$ & $78 \%$ & $100 \%$ & $87 \%$ \\
\hline Amikacin & $100 \%$ & $78 \%$ & $100 \%$ & $92 \%$ \\
\hline Gentamicin & $100 \%$ & $89 \%$ & $100 \%$ & $96 \%$ \\
\hline Ciprofloxacin & $92 \%$ & $78 \%$ & $100 \%$ & $90 \%$ \\
\hline Levofloxacin & $92 \%$ & $67 \%$ & $100 \%$ & $86 \%$ \\
\hline Cefepime & $92 \%$ & $89 \%$ & $100 \%$ & $94 \%$ \\
\hline Cotrimoxazole & $75 \%$ & $78 \%$ & $100 \%$ & $84 \%$ \\
\hline Erythromycin & $67 \%$ & $67 \%$ & $100 \%$ & $78 \%$ \\
\hline Clindamycin & $75 \%$ & $67 \%$ & $100 \%$ & $80 \%$ \\
\hline Vancomycin & $100 \%$ & $100 \%$ & $100 \%$ & $100 \%$ \\
\hline Tetracycline & $56 \%$ & $78 \%$ & $100 \%$ & $76 \%$ \\
\hline
\end{tabular}

Table 5: Antibiotic sensitivity pattern of Pseudomonas aeruginosa. $(\mathbf{n}=20)$

\begin{tabular}{|c|c|c|c|c|c|c|c|c|c|c|c|}
\hline Drugs & Ak & Gen & Cip & Le & CAZ & CPD & CPM & CPZ & IPM & AT & PTZ \\
\hline Pseudo & $76 \%$ & $50 \%$ & $50 \%$ & $32 \%$ & $12 \%$ & $8 \%$ & $60 \%$ & $22 \%$ & $96 \%$ & $50 \%$ & $92 \%$ \\
\hline
\end{tabular}

\section{Discussion}

The Microbial pathogens as well as their antibiotic resistance patterns may change from time to time and place to place. The emergence of antibiotic-resistant organisms, the increase in the frequency of nosocomial infections, and increasing number of immunecompromised patients have combined to keep pleural and ascitic fluid infections a common entity. ${ }^{4}$

In our study, $22 \%$ of samples gave culture positive result, which is in comparison to other studies, which were $30 \%, 31 \%$ positive results.

In our study the predominant organisms were Escherichia coli (33), NFGNB (27), followed by Pseudomonas (20), Klebsiella (19), Staphylococcus aureus (15), Citrobacter species (13), CONS (8), Enterococcus (4), Enterobacter (2), Streptococcus pyogenes (1).

The most common organism isolated from pleural fluid were Escherichia coli (16), NFGNB (10), Staphylococcus aureus (10), Pseudomonas (12). Our study findings correlates with the study of Rajani et $\mathrm{al}^{5}$ where predominant organism was E-coli followed by Acinetobacter. Our results are in contrast to studies of Sujatha et $\mathrm{al}^{6}$ and Evan et $\mathrm{al}^{11}$ where in E-coli, Klebsiella and Staphylococcus aureus were the most common isolates.

Our study highlights the emergence of aerobic gram negative bacteria as the predominant pathogens in empyema. A similar high rate of isolation of GNB from pleural fluid cultures was reported in India by Sonali $(88.4 \%),{ }^{8}$ Gupta S K et al $(84.6 \%),{ }^{9}$ Mohanty et al $(86.4 \%){ }^{10}$ Prior to the availability of antibiotics, S.pneumoniae and S.pyogenes accounted for most of the empyema cases. After the discovery and widespread use of antibiotics in the 1940s, Staphlococcus aureus succeeded S.pneumoniae and S.pyogenes as the major cause of empyema. Since the advent of beta-lactamase resistant semi-synthetic penicillins in the 1960 s, the incidence of staphylococcal empyema has decreased and infections due to aerobic GNB as the predominant pathogen has increased markedly. The isolation of aerobic GNB or multiple pathogens from pleural fluid is associated with a poor prognosis and indicates a more aggressive antimicrobial chemotherapy in contrast to the empyema caused by Gram positive pathogens.

Gram negative bacteria were more commonly isolated from Ascitic fluid than Gram positive bacteria. Among Gram negative bacteria NFGNB (13) was the most common isolate followed by E-coli (9), Klebsiella (9), Pseudomonas (9), which is in contrast to the study of Sujatha R et al, ${ }^{6}$ Arroyo et a ${ }^{12}$ and Chawla $\mathrm{P}^{13}$ which showed $E$-coli as the commonest organism. Our results show that $N F G N B$ is more prevalent in ascitic fluid samples in our geographical area. However further studies with more sample size has to be done to find the common etiological agents of ascitic fluid in our set-up.

Bacterial meningitis is being reported predominantly in adults in USA because of immunization practices adopted and also due to relative increase in frequency of noscomial meningitis. In North America and Europe because of the vaccine related decline in H.influenzae disease, Streptococcus pneumonia and Nesseriae meningitides remain important pathogens in Children and young adults. Group B streptococcus is the most common pathogen associated with meningitis in newborns. Listeria 
monocytogenes is also recognized as a significant cause of meningitis in newborns and the elderly in the United States. As compared to western studies, the relative incidence of meningitis caused by H.influenzae, Nessieriae meningitides and listeria is less in South East Asia.

On the contrary Gram Negative bacilli such as $E$ coli Klebsiella pneumonia and Pseudomonas aeruginosa are increasingly being reported in cases of meningitis especially among elderly and in patients with cirrhosis, diabetes and malignancies. ${ }^{14-16}$ Interestingly, similar findings was observed in our study where E-coli and Klebsiella species were commonly isolated from CSF followed by NFGNB and Staphylococcus aureus.

In our hospital very few pericardial fluid samples were received, only $1 \%$ compared to other body fluids. Out of 6 samples received only 4 samples showed growth. CONS, E-coli, Klebsiella, NFGNB were isolated which is in contrast to the studies of $\mathrm{H}$ Reuter et $\mathrm{al}^{17}$ where Staphylococcus aureus and Salmonella species were commonly isolated. Bacterial infections of pericardium are relatively uncommon; however they are much more likely to form purulent effusions and to proceed to cardiac tamponade or pericardial constriction. Purulent pericarditis is almost exclusively seen as a secondary infection in the patients with seriously underlying diseases such as AIDS and those undergoing hemodialysis, thoracic surgery, and chemotherapy. It is not typically a primary infection but rather almost exclusively a complication of an underlying infections. ${ }^{18,19}$

In our study, the Gram negative bacterial isolates were $100 \%$ sensitive to Carbapenems. Good sensitivity was also observed to Amikacin Gentamicin Cefepime and less sensitivity to Ciprofloxacin, Levofloxacin and Amoxyclav. They were relatively resistant to Ampicillin, Ceftriaxone, Cefotaxime and Cotrimoxazole. E.coli isolates showed highest resistance to Cephalosporins and ampicillin. Our findings are in agreement with Rajani Sharma ${ }^{5}$ wherein gram negative isolates were $100 \%$ sensitive to Carbapenems and E.coli was highly resistant to cephalosporins and fluoroquinolones. In Tullu MS et $\mathrm{al}^{20}$ study too, majority of the isolates were highly resistant to ampicillin and caphazolin which is in agreement with our study findings.

Gram positive isolates were $100 \%$ sensitive to Vancomycin and Linezolid. They showed good sensitivity to Gentamicin (96\%), Cefepime (94\%), Amikacin (92\%), Ciprofloxacin (87\%), Amoxyclav (87\%), Levofloxacin (86\%), Cotrimoxazole (84\%). They were resistant to Ampicillin. Our results are in agreement with Sujatha et al. ${ }^{6}$

Pseudomonas species were highly sensitive to Imipenem (96\%) and Piperacillin Tazobactum (92\%). They showed good sensitivity to Amikacin, Cefepime. They were less sensitive to Aztreonam, Ciprofloxacin, gentamicin. They were resistant to Ceftazidime, Cefpodoxime, Cefperazone and Levofloxacin. Our results are in agreement to Rajani Sharma et al. ${ }^{5}$ but in contrast to Sujatha $\mathrm{R}^{6}$ wherein they showed good sensitivity to Ciprofloxacin and Ceftazidime.

\section{Conclusion}

In the present study E.coli was the most common organism isolated from various body fluids. Significant numbers of both gram negative and gram positive bacteria were isolated from various body fluid samples. The high level of MDR strains were observed among gram negative isolates which calls for immediate attention of health care workers and policy makers for the prudent antibiotic use and thus limit the transmission of MDR bacteria in the hospital and community settings.

Hence, surveillance of the incidence, microbial profile and antibiotic resistance pattern of sterile body fluids infections in a particular population is an essential part for the selection of the most appropriate empiric antibiotic regimen which helps the clinicians to treat effectively and thus prevent morbidity and mortality associated with these infections.

\section{Limitations}

The culture positivity of sterile body fluids was relatively less as the etiology of sterile fluid infections also includes anaerobic bacteria and Viruses, which were not included in our study.

\section{References}

1. Hughes JG, Vetter EA, Patel R, Schleck CD, Harmsen S. (2001) Cultureb with BACTEC Peds Plus/F bottle compared with conventional methods for detection of bacteria in synovial fluid. J Clin Microbiol. 2001;39:4468-4471.

2. Daur AV, Klimak F, Cogo LL, Botao GD, Monteiro CL. (2006) Enrichment methodology to increase the positivity of cultures from body fluids. Braz J Infect Dis. 10:372373.

3. Forbes BA, Sahm DF, Weissfeld A (2007) Bailey and Scott's Diagnostic Microbiology (12th Edn.).Mosby Elsevier, St Louis, Missouri.

4. Alfageme I, Munoz F, Pena N, Umbria S. Empyema of the thorax in adults. Etiology, microbiologic findings, and management: Chest. 1993;103(3):839-43.

5. Sharma R, Anuradha, Nandini D. Bacteriological Profile and Antimicrobial Sensitivity pattern in Sterile body fluids from a tertiary Care Hospital. J Appl Microbiol Biochem. 2017,1:1.

6. Sujatha R, Pal N, Arunagiri D, Narendram D. Bacteriological profile and antibiotic sensitivity pattern from various body fluids of patients attending Rama Medical College hospital Kanpur. Int $J$ of Advances in Case Reports. 2015;2:119-124.

7. Clinical laboratory Standard Institute.2017. Performance standard for antimicrobial disk susceptibility tests. Approved standard $-20^{\text {th }}$ Ed. Supplement/M2A92006:26(1). 
8. Jain Sonali, Banavaliker J N, Empyema Thoracis: Bacteriological analysis of pleural fluid, IOSR-JDMS. 2013;3(6):46-51. ISSN: 2279-0853, ISBN:2279-0861.

9. Gupta S K, Kishan J, Singh S P, Review of one hundred cases of empyema thoracis; Indian J Chest Dis Allied Sci. 1989;3(1):15-20

10. Srujana Mohanty, Arti Kapil, Bimal K Das. Bacteriology of parapneumonic pleural effusions in an Indian hospital: Trop D. 2007;37(4):228-229.

11. Evans LT, Kim WR, Poterucha JJ, Kamath PS. Spontaneous bacterial peritonitis in asymptomatic outpatients with cirrhotic ascites. Hepatology. 2003;37:897-901.

12. Arroyo V, Bataller R, Gines P. (2000) Spontaneous bacterial peritonitis. Comprehensive Clinical Hepatology, Barcelona, Mosby. Pp: 10-7.

13. Chawla P, Kaur D, Chhina RS, Gupta V, Sharma D. Microbiological Profile of ascitic fluid in patients of cirrhosis in a tertiary care hospital in Northern India. Internat J Pharmac Res and Biosci. 2015;4:144-153.

14. Mani R, Pradhan S, Nagarathna S, Wasiulla R, Chandramukhi A. Bacteriological Profile of Community -Acquired Acute Bacterial Meningitis: A Ten Year Retrospective Study in a tertiary Neurocare in South India. IJMM. 2007;25(2):108-14.

15. Tang LM, Chen ST, Hsu WC, Lyu RK. Acute Bacterial Meningitis meningitis in adults: A hospital-based epidemiological study. QJM. 1999;92;719-25.
16. Schuchat A, Robinson K, Wenger JD, Harrison LH, Farley M, Reingold AL, Lefkowitz L, Perkins BA. Bacterial meningitis in the United States in 1995. Active Surveillance Team. N Eng J Med. 1997;337:970-76.

17. H. Reuter, L.J. Burgess and A.F. Doubell. Epidemiology of Pericardial effusion at large Academic hospital in South Africa. Epidemiol Infect, 2005;133(3):393-399.

18. Pankuweit S, Ristic AD, Seferovic PM, Maisch B. Bacterial pericarditis: diagnosis and management. Am J Cardiovasc drugs. 2005;5(2):103-12.

19. Keersmaekers T, Elshot SR, Sergeant PT. Primary bacterial pericarditis. Acta Cardiol. 2002;57(5):387-9.

20. Tullu MS, Deshmukh CT, Baveja SM. Bacterial profile and antimicrobial susceptibility pattern in catheter related nosocomial infections. J Postgrad Med. 1998;44:7-13.

How to cite this article: Harshika Y K, Shobha M. K. R, Patil A B, Smita N R. A study on bacteriological profile and antimicrobial resistance pattern from various body fluids of patients attending the tertiary care Hospital, KIMS, Hubli. Indian J Microbiol Res. 2018;5(4):530-534. 